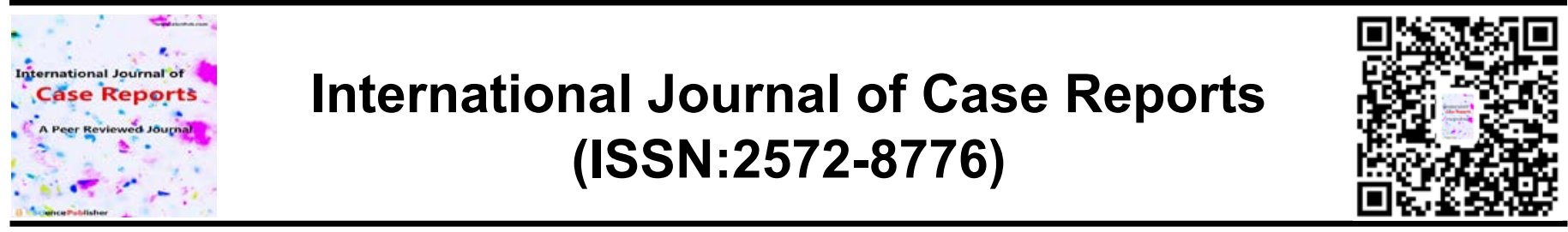

\title{
A Case Report Highlighting Some of the Difficulties Faced Diagnosing Appendicitis in Children
}

\author{
S. Lewis, A. Rasheed \\ Royal Gwent Hospital
}

\section{ABSTRACT}

Introduction: In England 12,000 children are diagnosed with appendicitis each year. The diagnosis of appendicitis is difficult especially in children. There are many other diagnoses which mimic appendicitis including Meckel's diverticulum, intussusception, gastroenteritis and mesenteric adenitis. Unfortunately there is no gold standard in the diagnosis of appendicitis and often clinicians are hesitant to submit a child to radiological investigations.

Presentation of the case: An eleven year old boy, of Bangladeshi origin, presented to the Royal Gwent Hospital, South Wales, with an acute onset of centralised abdominal pain and vomiting. Following a period of observation and re-examination his pain had localised to the right iliac fossa and it was decided on clinical grounds to perform a laparoscopy for suspected appendicitis. Intra-operative findings showed an intussusception of the small bowel secondary to a mucosal lesion. Histology showed high grade non-Hodgkin's lymphoma with a pattern suggestive of Burkitt's lymphoma.

Conclusion: The case highlights the importance of accurate diagnosis of appendicitis and the difficulties of managing abdominal pain in children. More needs to be done to develop a better criterion and judicial use of imaging to guide surgical intervention.

Keywords: Appendicitis, Alvarado, Ultrasound, Computer Topography, Laparoscopy
*Correspondence to Author:

S. Lewis and A. Rasheed

Royal Gwent Hospital

How to cite this article:

S. Lewis and A. Rasheed.A Case Report Highlighting Some of the Difficulties Faced Diagnosing Appendicitis in Children. International Journal of Case Reports, 2018 3:38

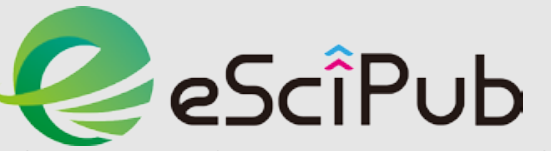
eSciPub LLC, Houston, TX USA. Website: http://escipub.com/ 


\section{Case-Report}

An eleven-year-old boy, of Bangladeshi origin, presented to the Royal Gwent Hospital (South Wales, United Kingdom) with a four hour history of acute central abdominal pain and vomiting. On examination the abdomen was tender over the umbilicus but soft with no concerning signs of peritonism. Blood tests showed an isolated elevated white blood cell count. The decision was made to actively observe by re-examining; six hours later, he was found to be tender in the right iliac fossa including over McBurney's point. The decision was made to proceed to a diagnostic laparoscopy for a presumptive diagnosis of acute appendicitis. However, intraoperatively he was found to have intussusception of the small bowel secondary to a mucosal lesion (see figure 1). The procedure was converted to a laparotomy and the intussuscepting point was resected, along with $20 \mathrm{~cm}$ of distal bowel (see figure 2) and a side to side anastomosis undertaken. Macroscopically a $2.5 \mathrm{~cm}$ firm polyp was excised and histology showed a high-grade non-Hodgkin's lymphoma with finding's suggestive of Burkitt's lymphoma. Postoperatively the young boy recovered without any complications. His care was transferred to another centre with specialist paediatric oncology treatment.

\section{Discussion}

In England 12,000 children (less than 18 years of age) undergo emergency appendicectomies with 4 in 5 managed in district general hospitals. For every diagnosis of appendicitis, four other children will present to secondary care with nonspecific abdominal pain ${ }^{1}$. Previously a child's history and clinical examination would form the decision as to whether a diagnostic laparoscopy is indicated; however, recent literature is leaning towards the addition of imaging investigations. Additionally more and more children are being treated conservatively with antibiotic therapy².

Appendicitis is difficult to diagnose but should not be delayed to reduce the risk of perforation and other possible complications. In children, there is no gold standard for the diagnosis of appendicitis. Children often struggle to describe their pain and symptoms, plus there are many other diagnoses which mimic appendicitis such as Meckel's diverticulum, intussusception, gastroenteritis and mesenteric adenitis. Although imaging is useful, it could involve significant ionising radiation to the child if CT scanning is selected in addition to the costs implications and the psychological impact on the child ${ }^{2,3}$

To try and aid diagnosis clinicians have tried to establish clinical predicative criteria. The two most validated include the Alvarado and Paediatric Appendicitis Score (PAS). The Alvarado score was initially developed in 1986 and directed towards the diagnosis of appendicitis in adults. The PAS score was reported by Samuel in 2002 and both include almost identical criteria ${ }^{4}$ (figure 3 ). A score of 1 3 is a strong predictive factor against appendicitis and a score of $8-10$ is highly suggestive of a positive diagnosis. The values 47 indicate moderate risk but are inconclusive of a diagnosis. Although such tools are useful, they still have their limitations and found to overdiagnose appendicitis in $32-35 \%$ of cases $^{3}$. Some clinicians are trying to develop better tools by combining the predictive criteria with ultrasonography leading to a higher diagnostic sensitivity and specificity of appendicitis ${ }^{4}, 5$. Ultrasound (USS) imaging would have been helpful in this case and would have diagnosed the intussusception preoperatively. USS is noninvasive, low risk and if performed by an experienced user, can diagnose appendicitis. However it is dependent on the operator, the location of the appendix and the patient's body habitus with the visualisation of the appendix, according to the literature, varying between 2$82 \%$. Although USS cannot conclusively diagnose appendicitis, it can aid the clinician in management hopefully reducing the number of CT scans (and radiation exposure) and the number of children undergoing unnecessary surgery ${ }^{6}$. 


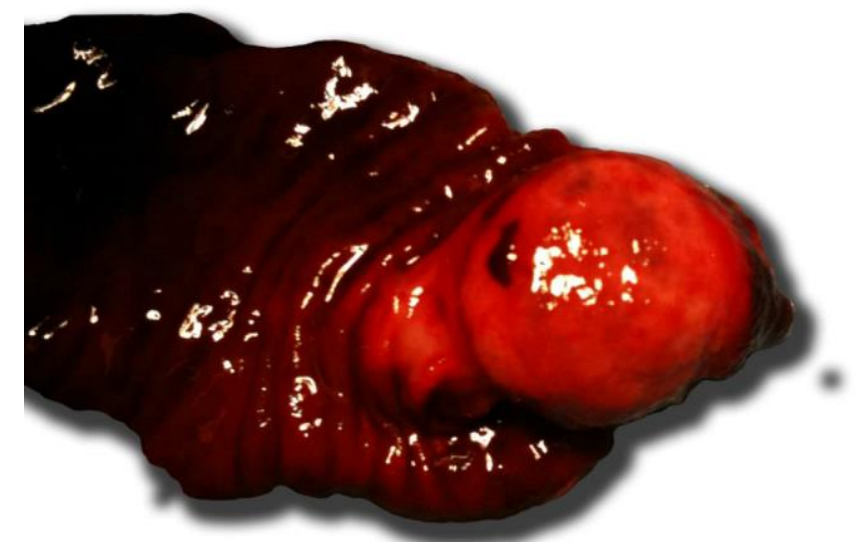

Figure 1- resected mucosal lesion causing the point of intussusception

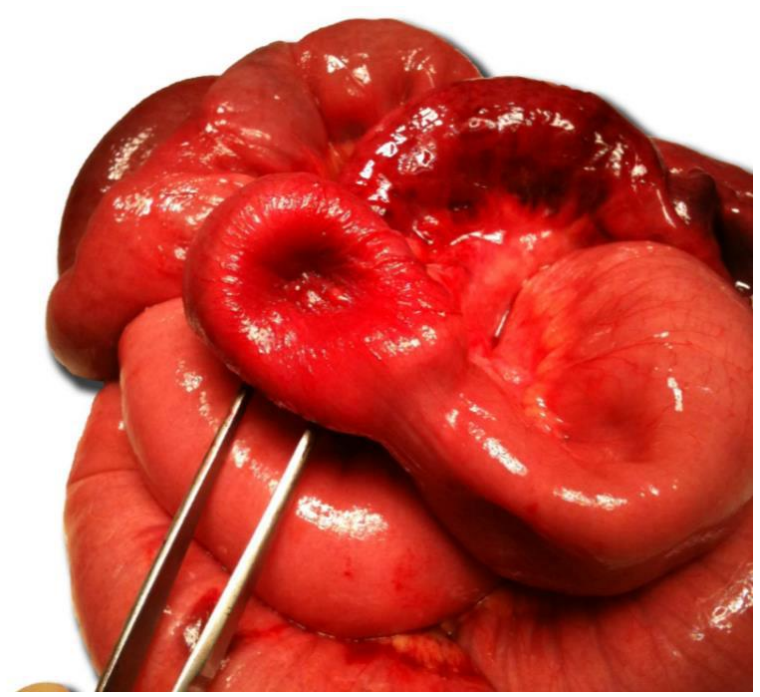

Figure 2- the intussuscepting point plus $20 \mathrm{~cm}$ of distal bowel resected

Management Algorithm for Patients with Clinically Suspected Appendicitis|

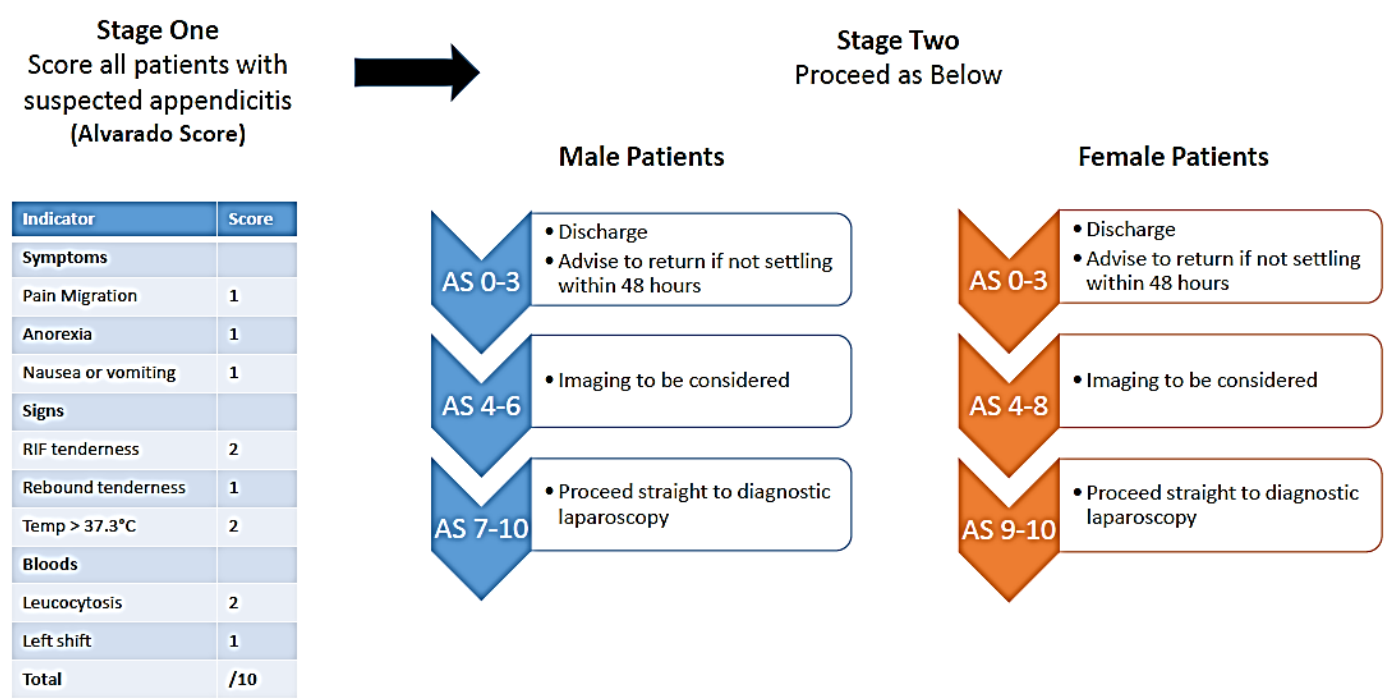

Figure 3 The flow chart represents our adopted management pathway based on Alvarado score 
The young boy described in our case had a successful outcome despite the difficulties posed by the unexpected finding. Although predictive criteria are limited, if used appropriately it can aid in the diagnosis of appendicitis and of value in guiding further management. This could also involve imaging or diagnostic laparoscopy.

\section{Conclusion}

The cause of abdominal pain can be difficult to diagnose in children due to the multiplicity of conditions mimicking each other. The most common surgical cause is acute appendicitis, however, there is no gold standard criterion for the diagnosis of

appendicitis. We need to streamline diagnosis and management of appendicitis in children by utilisation of validated predictive criteria and judicial use of imaging to guide therapeutic interventions and reduce the incidence of unnecessary surgery.

\section{Conflict of Interest}

I affirm that we have no financial affiliation or involvement with any commercial organization with direct financial interest in the subject or materials discussed in this manuscript.

\section{Author Contribution}

All authors have contributed and approved the final article.

\section{Ethical Approval}

Not applicable as it is a report of one case

\section{Consent}

Patient in the study design has been informed and aware about the study and that his interoperative images will be included. His identity, nevertheless, cannot be recognized from the case history and images.

\section{Funding: None.}

\section{References:}

1. Commissioning Guide: Paediatric Emergency Appendicectomy. British Association of Paediatric surgery; 2015

2. Nakayama DK, Examination of the Acute Abdomen in Children. Journal of Surgical
Education. 2016; 73(3): p548-552.

3. Kulik DM et al. Does the child have appendicitis? A systemic review of clinical prediction rules for children with acute abdominal pain. Journal of Clinical Epidemiology. 2012; 66(1): p95-104.

4. Saucier $A$ et al. Prospective Evaluation of a Clinical pathway for Suspected Appendicitis. Paediatrics. 2014; 133(1)

5. Toprak $\mathrm{H}$ et al. Integration of ultrasound findings with Alvarado score in children with expected appendicitis. Paediatrics International. 2014; 56(1):p95-99.

6. Henning F, Gfroerer S, Rolle U. Systemic review shows that pathological lead points are important and frequent in intussusception and are not limited to infants. ACTA Paediatrica - Nurturing the child. 2016; 105(11): 1275-1279.

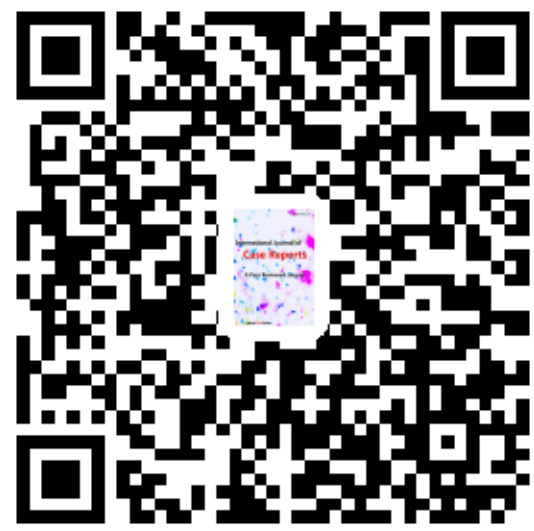

IJCR: http://escipub.com/international-journal-of-case-reports/ 